\title{
Monitoring therapy in canine malignant lymphoma and leukemia with serum thymidine kinase 1 activity - evaluation of a new, fully automated non-radiometric assay
}

\author{
HENRIK P. VON EULER ${ }^{1}$, PATRICIO RIVERA ${ }^{1}$, ANNE-CHARLOTTE ARONSSON ${ }^{2}$, \\ CHRISTER BENGTSSON ${ }^{3}$, LARS-OLOF HANSSON ${ }^{3}$ and STAFFAN K. ERIKSSON ${ }^{4}$ \\ ${ }^{1}$ Center of Clinical Comparative Oncology $\left(\mathrm{C}_{3} \mathrm{O}\right)$, Department of Clinical Sciences, Faculty of Veterinary Medicine and \\ Animal Science, Swedish University of Agricultural Sciences (SLU), P.O. Box 7054, S-750 07 Uppsala; ${ }^{2}$ DiaSorin AB, \\ P.O. Box 20196, S-161 02 Bromma; ${ }^{3}$ Akademiska Laboratory, Uppsala University Hospital, S-751 85 Uppsala; \\ ${ }^{4}$ Department of Anatomy, Physiology and Biochemistry, Swedish University of Agricultural Sciences (SLU), \\ P.O. Box 575, S-751 23 Uppsala, Sweden
}

Received September 9, 2008; Accepted October 13, 2008

DOI: 10.3892/ijo_00000175

\begin{abstract}
Thymidine kinase 1 (TK), which is involved in the synthesis of DNA precursors, is only expressed in S-G2 cells. Serum TK levels correlate to the proliferative activity of tumor disease. Determinations of TK levels have so far relied on radio enzyme assay (REA) and experimental ELISA methods, which have limited the clinical use of this biomarker, although recent studies in dogs with malignant lymphoma (ML) demonstrate its wide potential. A non-radiometric method based on a competitive immunoassay with specific anti-3'-azido-deoxythymidine monophosphate (AZTMP) antibodies has been further developed into the fully automated Liaison TK assay (DiaSorin). Sera from healthy dogs $(n=30)$, and dogs with leukemia (LEUK) $(n=35)$, ML $(n=84)$, nonhematological tumors $(\mathrm{n}=50)$, and inflammatory disease $(n=14)$ were tested using both methods. Lymphoma and LEUK samples were available before and during chemotherapy. The coefficients of variation for the Liaison TK assay in this study were 6.3 and $3.4 \%$ (low/high TK, respectively), and the correlation between TK REA (X) and the Liaison TK assay (Y) was $y=0.9203 x+1.3854\left(R^{2}=0.9501\right)$. The TK1 levels measured during chemotherapy gave very clear differences between dogs in complete remission and dogs out of remission.
\end{abstract}

Correspondence to: Dr Henrik von Euler, Center of Clinical Comparative Oncology $\left(\mathrm{C}_{3} \mathrm{O}\right)$, Department of Clinical Sciences, Faculty of Veterinary Medicine and Animal Science, Swedish University of Agricultural Sciences (SLU), P.O. Box 7054, SE-750 07 Uppsala, Sweden

E-mail: henrik.von.euler@kv.slu.se

Key words: thymidine kinase 1, tumor marker, prognosticate, canine, hematological malignancy, chemotherapy
A Tukey-Kramer analysis showed that all LEUKs and MLs out of remission differed significantly from the other groups. The Liaison TK assay showed high precision, high sensitivity and a good correlation to the TK REA. The Liaison TK assay provides valuable clinical information in the treatment and management of canine LEUK and ML, with a potential to be further validated in human trials.

\section{Introduction}

Serum tumor markers are used to obtain prognostic information and to monitor tumor disease both in human and in veterinary medicine. Thymidine kinase 1 (TK1) is a deoxyribonucleic acid (DNA) precursor enzyme that is only found in proliferating cells and is one of the key markers for S-phase and G2 cells in cell biology. A large amount of biochemical information is now available concerning the structure-function relationships of TK1, which have been of considerable interest for the antiviral and anti-cancer chemotherapy field (1-3). The cell cycle and tissue regulation of TK1 messenger ribonucleic acid (mRNA) and protein, which depends on transcriptional, translational, and posttranslational processes, has also been studied in great depth (3-5).

A stable serum form of TK1 was identified already in 1980 and serum TK (s-TK) levels serve as a biomarker in many different malignant diseases, e.g., Hodgkin's and non-Hodgkin's lymphoma (NHL), acute myeloid leukemia (AML), lymphocytic leukemia (LL), and chronic LL (6-8). One report describes s-TK activity and lactate dehydrogenase (LDH) activity in dogs with malignant lymphoma (ML) (9). More recently, s-TK REA determinations including the use of a non-radiometric assay were shown to provide prognostic and monitoring information regarding disease progression in canine ML $(10,11)$.

A non-radiometric TK enzyme-linked immunosorbent assay (ELISA) was developed by Öhrvik et al, who used a thymidine analog, 3'-azido-2'-deoxythymidine (AZT), as 
substrate (12). The new TK assay could be used for estimating TK1 activity in dogs with ML with the same accuracy as reported for the TK REA (10).

Naturally occurring cancers in pet dogs and humans share many features, including histological appearance, tumor genetics, molecular targets, biological behavior, and response to conventional therapies (13) (Fig. 1). The complete dog genome sequence was recently published, showing high homology with the human sequence (14). In 2007, the power of using the dog genome to map genetic risk factors shared with humans become evident $(15,16)$.

Studying dogs with cancer provides a valuable perspective that is distinct from that generated by the study of human or rodent cancers alone (17-20). The value of studying dogs with cancer has increasingly been recognized in the field of cancer research for the identification of cancer-associated genes, the study of environmental risk factors, understanding tumor biology and progression, and, perhaps most importantly, the evaluation and development of novel cancer therapeutics (13). Malignant lymphoma in dogs in particular has been shown to share morphological gene expression patterns and clinical similarities with human NHL (21-23).

In the present study, we used a fully automated version of the AZT assay provided by DiaSorin, Inc. (Stillwater, MN, USA), and developed for the Liaison system using an isoluminol-AZTMP conjugate (24). In total, 183 sera from dogs with leukemia (LEUK), ML, non-hematological malignancies, and inflammatory conditions were tested using the Liaison TK assay and TK REA. In addition, 30 healthy dogs were included as controls. The result showed a high correlation and high clinical benefit of TK as a disease marker.

\section{Materials and methods}

All treatments and sampling of dogs were approved by the Swedish Animal Ethics Committee (no. C122/0).

Blood samples $(n=213)$ (stored at $\left.-20^{\circ} \mathrm{C}\right)$ were analyzed from a serum sample bank containing specimens from dogs mainly with ML, sampled between June 2006 and December 2007. Histologic/cytological confirmation of diagnosis was procured in all tumors. Each dog tumor was staged according to the classification scheme of the World Health Organization (25). Diagnostic methods employed varied depending on the histologic type of tumor, the anatomic location of the tumor, and the clinical status of the dog. These included, but were not limited to, physical examination, complete blood count (CBC), serum biochemical analysis, urinalysis, and radiographic studies. In total, 84 samples from dogs with ML, 35 LEUK samples, 50 samples from dogs with non-hematologic neoplasias, and 14 samples from dogs with inflammatory conditions were analyzed. In addition, 30 healthy dogs were included as normal controls.

Since new serum samples were added along the course of chemotherapy, several of the samples eventually proved to come from the same individual but to have been taken on different occasions. The total number of animals used in this study was 95 . The subjects were 30 normal dogs, 7 dogs with mast cell tumors, five with osteosarcomas, 6 with soft tissue sarcoma, 2 with mammary carcinomas, 2 with thyroid carcinomas, and 2 with squamous cell carcinomas, 1 dog with transitional cell carcinoma of the bladder, 1 with insulinoma, 29 with ML, and 10 with LEUK. In total, the number of samples analyzed was 213 . Originally, 230 samples were blinded and randomly collected from the serum bank. Seventeen samples were excluded due to insufficient volume for a second TK REA as a dilution was needed (TK >80 U/l, $\mathrm{n}=7$ ), or because they were from an incorrect species (feline, $\mathrm{n}=10$ ). The analyst had no knowledge of the origin of each sample.

The mean age of animals was 7.6 (range 1-12) years, and the group consisted of 48 males and 47 females. Twenty-two breeds are represented in this study. The most frequently represented breeds were Rottweilers $(10 / 95 ; 11 \%)$, followed by dogs of mixed breed $(9 / 95 ; 9 \%)$, and Golden Retrievers and English Springer Spaniels (with 8/95, 8\%, each). The normal controls were health-monitored dogs donating samples to the dog genome bank at SLU.

Malignant lymphomas and AMLs were treated with a doxorubicin-based multi-agent protocol (ADRIA-Plus) (26), while CMLs were treated with Lomustine (CCNU) orally (70 $\mathrm{mg} / \mathrm{m}^{2}$ q 21 days).

For the TK, levels in serum were determined using the TK Prolifen assay (DiaSorin AB, Bromma, Sweden) (6). All TK values are given as units per liter (U/l). The normal cutoff value in dogs for this REA is $<7$ U/1 (2 standard deviations (SDs) above the mean) (10).

The starting point for development of the Liaison TK assay and its first prototype is described in Sander et al, Clin Chem 49: abs. 6, 2003) and Öhrvik et al (12), but the fully developed and commercially available method was introduced in 2007 by DiaSorin, Inc., Stillwater, MN, USA (24). The principle is that TK1 present in the patient sample catalyzes phosphorylation of 3 -azido-thymidine (AZT) to the corresponding 5'-monophosphate, AZTMP. In the second step, the produced AZTMP competes with AZTMP conjugated to isoluminol (AZTMP-ABEI) for specific antibodies bound to magnetic beads. Free AZTMP-ABEI is removed by washing steps and then the amount of bound AZTMP-ABEI is determined by chemiluminesence.

The TK activities were analyzed both before and after log transformation (natural $\log$ ) because of skewed distribution. Group comparisons were performed by means of analysis of variance (ANOVA). A plot of the difference against the average of the standard (REA) and new measurements (Liaison) was performed using the Bland-Altman method (bivariate fit of difference) (27). In addition, Spearman's rank correlation analysis was performed to assess the relationship between the different assays. Comparison between healthy controls, inflammatory disease, other tumors, ML, and LEUK was done using one-way analysis (Tukey-Kramer honestly significant difference, HSD), due to unequally sized groups with unequal variances. The $\alpha$ level was set to 0.05 . Statistical calculations were performed using the JMP ${ }^{\circledR}$ package, version 7.0 (SAS Institute, Inc., Cary, NC, USA). Values of $\mathrm{P}<0.05$ were considered indicative of statistical significance.

\section{Results}

The TK activity ranged from 0.3 to $336 \mathrm{U} / 1$ in the TK REA analysis, and from 0.2 to $263 \mathrm{U} / 1$ in the Liaison TK assay. 


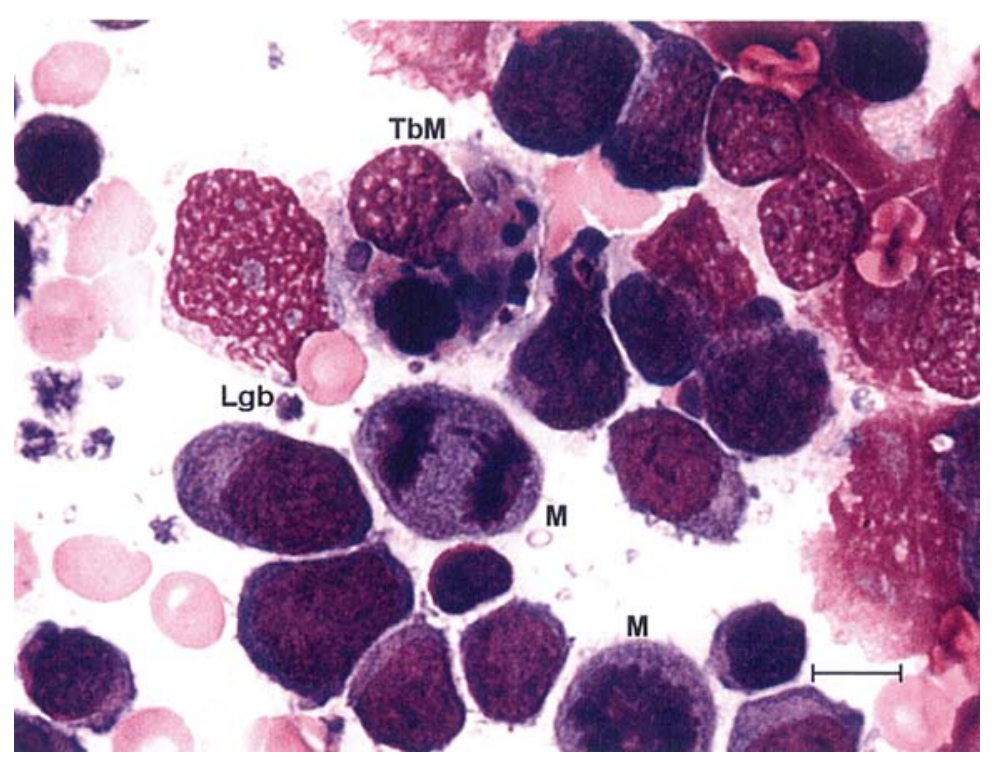

Figure 1. A fine-needle aspirate from a canine lymphoblastic lymphoma. Note the mitoses (M), lymphoglandular bodies (Lgb), and tingible-body macrophage $(\mathrm{TbM})$, all common features of malignant lymphoma (ML), also in humans. Cytology is an accurate diagnostic tool for ML, but does not provide reliable prognostic information. Serum biomarkers, such as serum thymidine kinase 1 (s-TK), can here act as a valuable complement in staging and prognosis. MayGrunewald-Giemsa staining, size bar $10 \mu \mathrm{m}$.

Table I. Thymidine kinase 1 (TK) activity measured using the Liaison TK assay. ${ }^{\mathrm{a}}$

\begin{tabular}{lccc}
\hline & Mean & Median & Range \\
\hline Normal $(\mathrm{n}=30)$ & 2.3 & 1.7 & $0.4-6.1$ \\
Inflammatory disease $(\mathrm{n}=14)$ & 2.1 & 2.0 & $0.7-3.0$ \\
Other tumors $(\mathrm{n}=50)$ & 3.3 & 1.7 & $0.5-16.2$ \\
ML in complete remission $(\mathrm{n}=56)$ & 3.0 & 18.6 & $0.5-7.0$ \\
ML out of remission $(\mathrm{n}=28)$ & 22.0 & 24.0 & $7.2-66.3$ \\
LEUK in remission $(\mathrm{n}=11)$ & 21.6 & 57.3 & $12.8-26.4$ \\
LEUK out of remission $(\mathrm{n}=24)$ & 88.1 & $30.7-263.0$ \\
\hline
\end{tabular}

anflammatory diseases, non-hematological neoplasias, and lymphomas in complete remission (CR) have TK activity levels not significantly different from those of healthy dogs, while the mean TK activity in malignant lymphomas (MLs) out of remission and leukemias (LEUKs) is 10- to 20 -fold higher than the means of the other groups. None of the dogs with LEUK reached CR, only partial remission. This shows the clinical benefit of monitoring hematological neoplasias with serum TK (s-TK), as well as indicating a high specificity of the Liaison assay.

There was a significant linear correlation $(\mathrm{P}<0.001)$ between the TK REA and the Liaison TK values (Fig. 2A). The simple linear regression analysis of the TK REA and Liaison TK $(\mathrm{U} / \mathrm{l})$ was Liaison $\mathrm{TK}=0.92 \mathrm{x}$ TK REA $+1.39, \mathrm{R}^{2}=0.9501$. Interassay variation was $5.6 \%$ and $3.5 \%$ for low $(5.3 \mathrm{U} / \mathrm{l})$ and high (19.2 U/l) control, respectively, in the TK REA. The total coefficients of variation for the Liaison TK assay in this study were 6.3 and $3.4 \%$, respectively, at the levels of 11.0 and $55.6 \mathrm{U} / 1$ (Fig. 3).

According to a bivariate fit of the difference performed, using the Bland-Altman method, there was no significant bias between the TK REA and the Liaison TK and the difference between the methods did not increase with higher values (Fig. 2B). Normal dogs had a mean of 2.3 and median of 1.7 (range 0.4-6.1) U/1, giving a normal cutoff of 5.5 U/1 in the Liaison assay. These results are similar to those reported by Fenske et al with samples from human patients with hematologic malignancies (24).

Serum TK activity was followed during chemotherapy in dogs with ML and LEUK. Initial high values were always present and reflected severity (clinical stage) of the disease. In dogs achieving remission (complete and/or partial), the TK activity returned to normal (CR) or decreased significantly compared with the pretreatment value (Fig. 2C). In dogs in whom the disease relapsed, the TK activity reflected an increase in tumor burden and predicted a fatal outcome. In dogs where remission was re-induced, TK activity returned to normal levels.

Thymidine kinase 1 activity is summarized in Table I. The table shows that inflammatory diseases, non-hemato- 
A

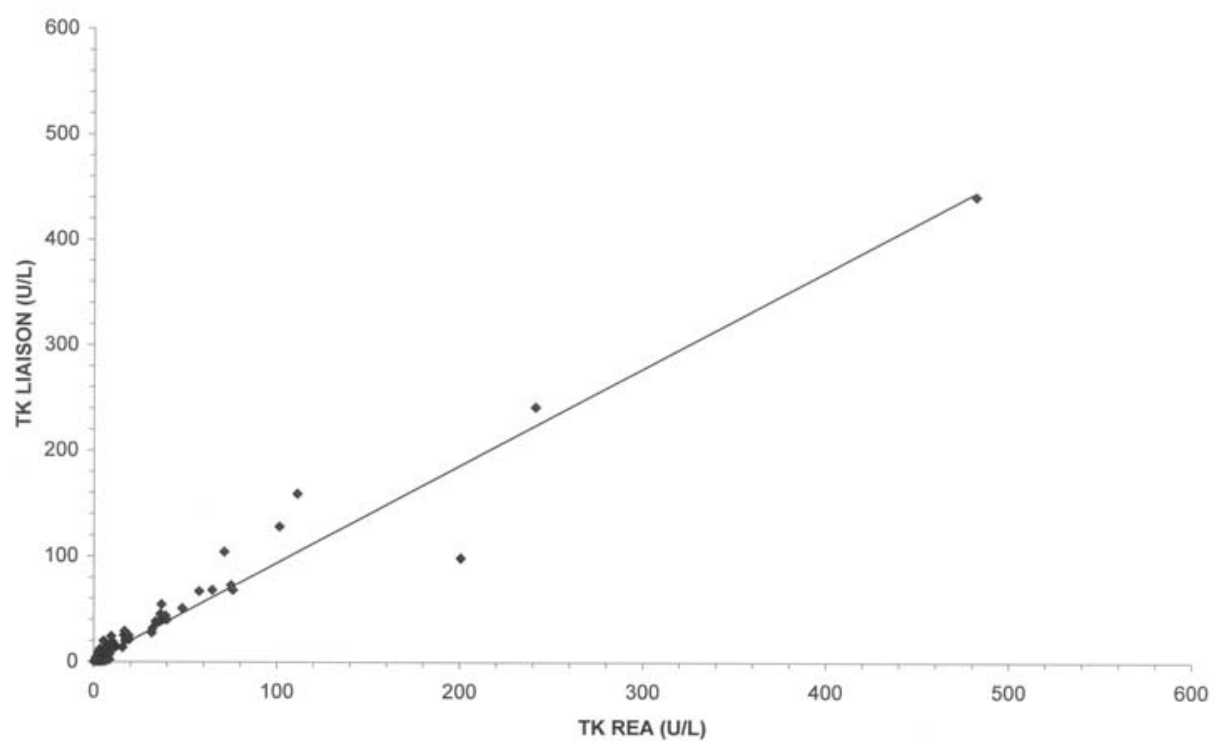

B

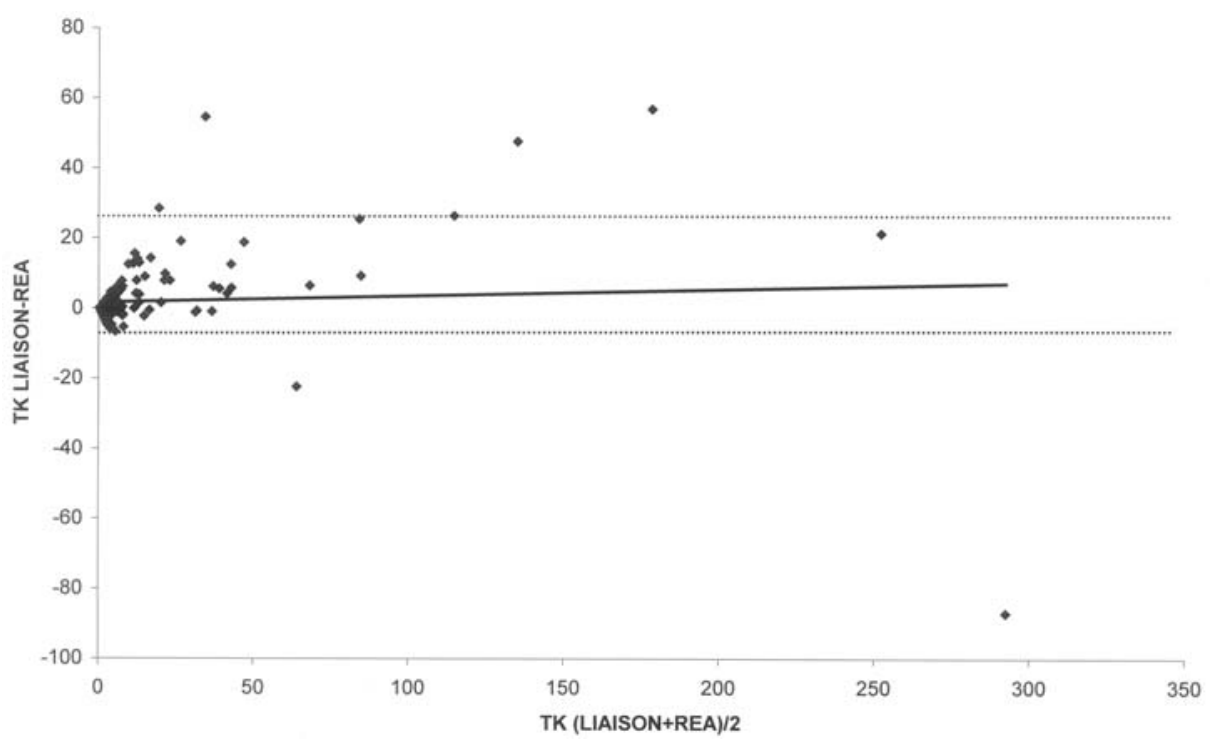

C

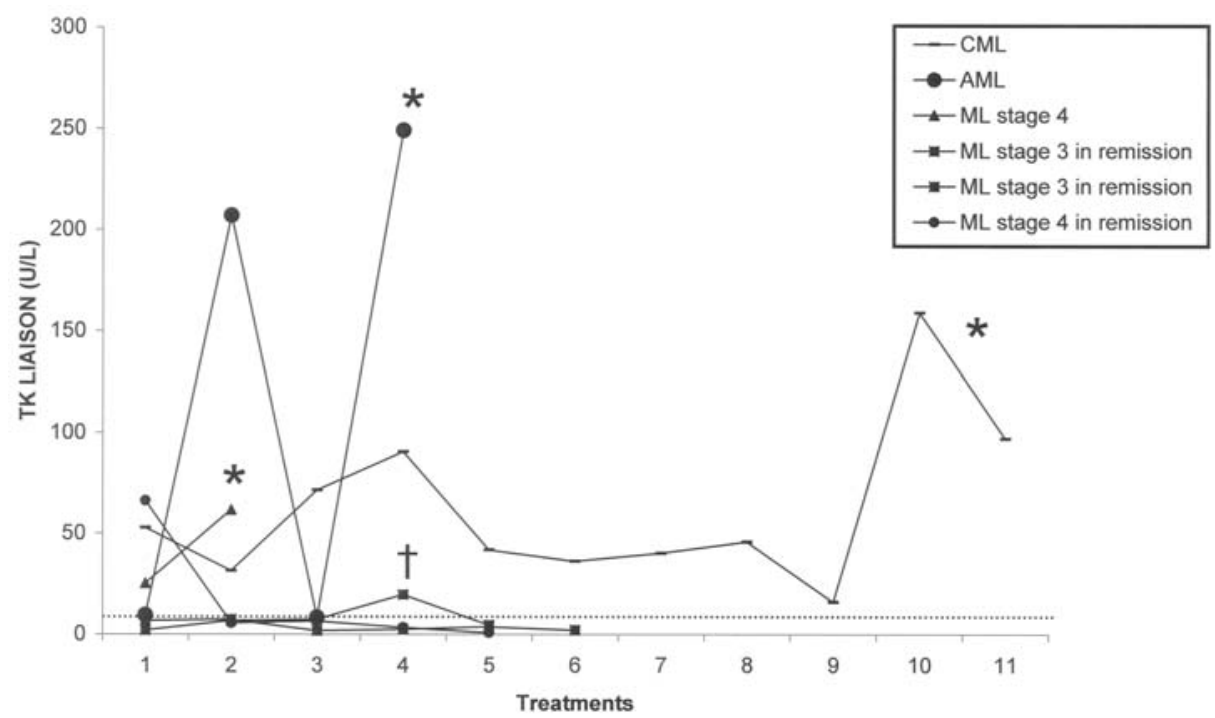

Figure 2. A, Comparison of the thymidine kinase 1 radio enzyme assay (TK REA) and Liaison TK measurements of 183 sera from malignant lymphoma (ML) patients $(n=119)$, and from dogs with non-hematological malignancies $(n=50)$ and inflammatory conditions ( $n=14)$. The correlation between the TK REA $(\mathrm{X})$ and the Liaison TK assay $(\mathrm{Y})$ was $\mathrm{y}=0.9203 \mathrm{x}+1.3854\left(\mathrm{R}^{2}=0.9501\right)$. B, Bland-Altman curve describing the relation between the TK REA and the Liaison TK assay. The figure shows the difference against the average of test and standard measurements, with $95 \%$ limits of agreement (broken lines) and a regression line. C, Serum thymidine kinase 1 (s-TK) activity measured with Liaison TK in various stages of disease in dogs with chronic myeloid leukemia (CML), acute myeloid leukemia (AML), and malignant lymphoma (ML). ${ }^{*}$ Dogs that failed to reach remission and succumbed to the disease; ${ }^{\dagger}$ ogs that had relapsed and which after rescue therapy again reached CR. The dotted line reflects the normal cutoff value. 


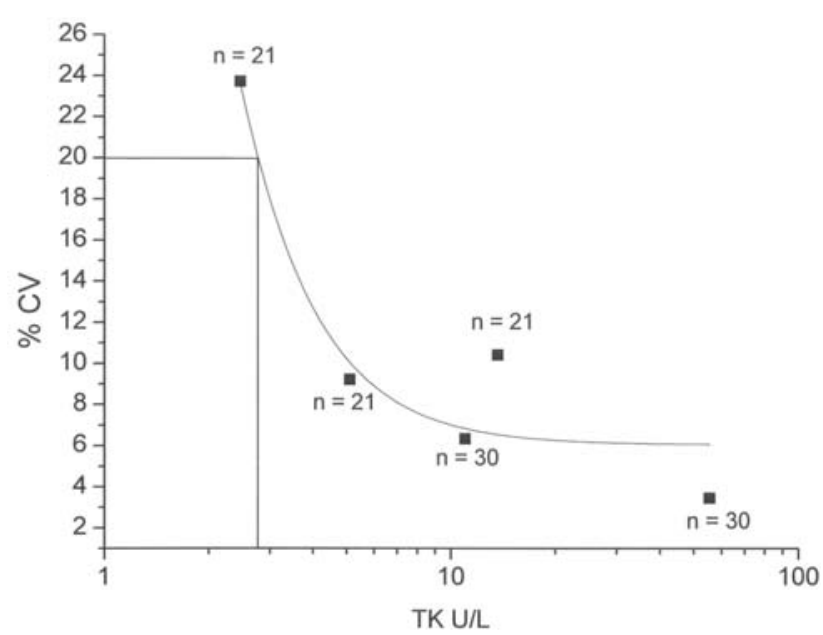

Figure 3. Precision profile for the Liaison TK assay.

logical neoplasias, and lymphomas in CR have TK activity levels not significantly different from those of healthy dogs. By contrast, the mean TK activity was 22.0 and $88.1 \mathrm{U} / 1$, respectively, in ML and LEUKs out of remission, which is 10- to 20-fold higher than the means of the other groups. A few LEUKs reached partial remission $(n=11)$ and had a mean TK of 21.6 U/1 (range 12.8-26.4), significantly different from the group of LEUK out of remission. The three nonhematological tumor samples with TK activity above normal were two hemangiosarcomas and one high-stage squamous cell carcinoma. The ML group was split into dogs in complete remission $(\mathrm{CR})(\mathrm{n}=56)$ or not $(\mathrm{n}=28)$. The mean for lymphomas in CR was 3.0 (range 0.5-7.0), while for dogs out of remission, it was 22.0 (range 7.2-66.3). After log transformation of TK values, a Tukey-Kramer analysis was performed. There was no significant difference between normal controls, inflammatory disease, other tumors, and MLs in CR, while the LEUKs (none reaching CR) and MLs out of remission were significantly different from the other groups with the $\alpha$ level set at 0.05 (Table I).

\section{Discussion}

This is the first clinical report investigating a convenient, fully automated, and non-radioactive method, the Liaison TK assay, and comparing it with the well-established TK REA.

As previously reported, analysis of s-TK is beneficial in the prognosis of, and monitoring disease progression in, canine ML, however, the method used in that study (10), was radioisotope-based, expensive, and not widely available. The Liaison TK assay evaluated in this study compares favorably with the TK REA and is most likely more TK1specific (i.e., more S-phase-specific), which makes it even more suitable for identifying tumor cell proliferation.

As shown previously by von Euler et al $(10,11)$ investigating both TK REA and TK ELISA methods, the Liaison TK values correlate with type and stage of disease, giving a very clear margin between healthy dogs and dogs with hematologic malignancies (Table I). Therefore, measurements of s-TK are a sensitive means to identify patients that should benefit from early or altered chemotherapy. It appears also to be particularly suited for early detection of relapse, leading to more efficient rescue therapy.

The sensitivity of the Liaison TK assay, which has a detection limit of $<1 \mathrm{U} / 1$ and which, in the present study, showed TK activity ranging from 0.2 to $263 \mathrm{U} / 1$, is adequate for measuring the TK activity present in sera from all individuals. The precision of the assay also makes it suitable for clinical management. The fact that the assay is now converted to a fully automated system means that it can easily be used in a standardized clinical setting. Decisions on treatment planning and prognostic information, which are important both for the veterinarian/physician and for the patient, can now be presented more rapidly and would certainly be of psychological importance for the client, as well as providing objective evidence of treatment outcome and of possible need for altered treatment regimes or further diagnostic procedures. In particular, the field of veterinary oncology is helped by objective guidance on whether to proceed with chemotherapy or not. Independent, objective markers for prognosis, monitoring of response, and determination of minimal residual disease are of great value in human clinical oncology, as well as in veterinary medicine, where the objective is to ensure optimal care, with regard to both animal welfare and the owner's money. Modern digital imaging techniques with computed tomography (CT) and magnetic resonance imaging (MRI) to provide the clinician with information of the tumor burden are increasingly being used also in veterinary medicine. However, if a continuous measurement of s-TK could assist the clinician in optimizing the use of other diagnostics and treatment planning, this would improve quality of life for the dogs, as well as the treatment response, and the use of dog owners' money, besides optimizing insurance payments.

Thymidine kinase is present in virtually all mammals (28). Although initially developed for human purposes, both the TK REA and the Liaison TK assay are species-independent, since they measure enzyme activity. This warrants further investigations in veterinary medicine.

To our knowledge, the Liaison TK assay is the first nonradiometric assay that is able to accurately measure s-TK1 activity in dogs, being fully automated for large-scale use. This assay may enable full exploitation of s-TK1 as a tumor marker. The study shows that the Liaison TK assay is a technically accurate and reliable method to determine TK1 activity in sera from dogs with ML. Therefore, the Liaison TK assay will most likely be of value, in the clinical setting, in management of dogs with ML.

\section{Acknowledgements}

Professor Jens Häggstrom is acknowledged for statistical consultation. The authors appreciate the financial support from Linnea and Axel Ericsson's foundation and the AGRIA pet insurance research foundation. S.E. received support for the study from the Swedish Research Council. Parts of this work were presented as an oral scientific research communication at the XI International Symposium on Biology and Clinical Usefulness of Tumor Markers in Barcelona (Spain), 21st -24th February 2007. 


\section{References}

1. Welin M, Kosinska U, Mikkelsen NE, et al: Structures of thymidine kinase 1 of human and mycoplasmic origin. Proc Natl Acad Sci USA 101: 17970-17975, 2004.

2. Al-Madhoun AS, Tjarks W and Eriksson S: The role of thymidine kinases in the activation of pyrimidine nucleoside analogues. Mini Rev Med Chem 4: 341-350, 2004.

3. Eriksson S, Munch-Petersen B, Johansson K and Eklund $H$ : Structure and function of cellular deoxyribonucleoside kinases. Cell Mol Life Sci 59: 1327-1346, 2002.

4. Ke PY and Chang ZF: Mitotic degradation of human thymidine kinase 1 is dependent on the anaphase-promoting complex/ cyclosome-CDH1-mediated pathway. Mol Cell Biol 24: 514-526, 2004.

5. Hengstschlager M, Knofler M, Mullner EW, Ogris E, Wintersberger E and Wawra E: Different regulation of thymidine kinase during the cell cycle of normal versus DNA tumor virus-transformed cells. J Biol Chem 269: 13836-13842, 1994.

6. Gronowitz JS, Hagberg H, Kallander CF and Simonsson B: The use of serum deoxythymidine kinase as a prognostic marker and in the monitoring of patients with non-Hodgkin's lymphoma. $\mathrm{Br}$ J Cancer 47: 487-495, 1983.

7. Hallek M, Wanders L, Strohmeyer S and Emmerich B: Thymidine kinase: a tumor marker with prognostic value for non-Hodgkin's lymphoma and a broad range of potential clinical applications. Ann Hematol 65: 1-5, 1992.

8. Poley S, Stieber P, Nussler V, Pahl H and Fateh-Moghadam A: Serum thymidine kinase in non-Hodgkin lymphomas with special regard to multiple myeloma. Anticancer Res 17: 3025-3029, 1997.

9. Nakamura N, Momoi Y, Watari T, Yoshino T, Tsujimoto H and Hasegawa A: Plasma thymidine kinase activity in dogs with lymphoma and leukemia. J Vet Med Sci 59: 957-960, 1997.

10. Von Euler H, Einarsson R, Olsson U, Lagerstedt AS and Eriksson S: Serum thymidine kinase activity in dogs with malignant lymphoma: a potent marker for prognosis and monitoring the disease. J Vet Intern Med 18: 696-702, 2004.

11. Von Euler HP, Ohrvik AB and Eriksson SK: A non-radiometric method for measuring serum thymidine kinase activity in malignant lymphoma in dogs. Res Vet Sci 80: 17-24, 2006.

12. Öhrvik A, Lindh M, Einarsson R, Grassi J and Eriksson S: Sensitive nonradiometric method for determining thymidine kinase 1 activity. Clin Chem 50: 1597-606, 2004.

13. Paoloni M and Khanna C: Translation of new cancer treatments from pet dogs to humans. Nat Rev Cancer 8: 147-156, 2008.

14. Lindblad-Toh K, Wade CM, Mikkelsen TS, et al: Genome sequence, comparative analysis and haplotype structure of the domestic dog. Nature 438: 803-819, 2005.
15. Karlsson EK, Baranowska I, Wade CM, et al: Efficient mapping of mendelian traits in dogs through genome-wide association. Nat Genet 39: 1321-1328, 2007.

16. Salmon Hillbertz NH, Isaksson M, Karlsson EK, et al: Duplication of FGF3, FGF4, FGF19 and ORAOV1 causes hair ridge and predisposition to dermoid sinus in Ridgeback dogs. Nat Genet 39: 1318-1320, 2007.

17. Vail DM and MacEwen EG: Spontaneously occurring tumors of companion animals as models for human cancer. Cancer Invest 18: 781-792, 2000.

18. Knapp DW and Waters DJ: Naturally occurring cancer in pet dogs: important models for developing improved cancer therapy for humans. Mol Med Today 3: 8-11, 1997.

19. Hahn KA, Bravo L, Adams WH and Frazier DL: Naturally occurring tumors in dogs as comparative models for cancer therapy research. In Vivo 8: 133-143, 1994.

20. MacEwen EG: Spontaneous tumors in dogs and cats: models for the study of cancer biology and treatment. Cancer Metastasis Rev 9: 125-136, 1990.

21. Von Euler H, Khoshnoud R, He Q, et al: Time-dependent RNA degradation affecting cDNA array quality in spontaneous canine tumours sampled using standard surgical procedures. Int J Mol Med 16: 979-985, 2005.

22. Sueiro FA, Alessi AC and Vassallo J: Canine lymphomas: a morphological and immunohistochemical study of 55 cases, with observations on p53 immunoexpression. J Comp Pathol 131: 207-213, 2004.

23. Teske E: Canine malignant lymphoma: a review and comparison with human non-Hodgkin's lymphoma. Vet Q 16: 209-219, 1994.

24. Fenske J, Tuttle A, Olson G, Aronsson A-C and Schmidt J: Development of an automated non-radiometric assay for the thymidine kinase on the Liaison analyzer-Technical and Clinical evaluation. Tumor Biol 28 (Suppl. 1): 56, 2007.

25. Owen LN: Ch 9. Lymphoid and haematopoietic tissues (including lymphosarcoma of skin). In: TNM Classification of Tumours in Domestic Animals. Owen LN (ed.) World Health Organization, Geneva, pp46-47, 1980.

26. Piek CJ, Rutteman GR and Teske E: Evaluation of the results of a L-asparaginase-based continuous chemotherapy protocol versus a short doxorubicin-based induction chemotherapy protocol in dogs with malignant lymphoma. Vet Q 21: 44-49, 1999.

27. Bland JM and Altman DG: Comparing methods of measurement: why plotting difference against standard method is misleading. Lancet 346: 1085-1087, 1995.

28. Arner ES and Eriksson S: Mammalian deoxyribonucleoside kinases. Pharmacol Ther 67: 155-186, 1995. 